\title{
Application of Multilayer Perceptron with Backpropagation Algorithm and Regression Analysis for Long-Term Forecast of Electricity Demand: A Comparison
}

\author{
D.B.L. Bong ${ }^{1}$, J.Y.B. Tan ${ }^{1}$, K.C. Lai ${ }^{1}$ \\ ${ }^{1}$ Universiti Malaysia Sarawak \\ bbldavid@feng.unimas.my
}

\begin{abstract}
Having an accurate forecast of future electricity usage is vital for utility companies to be able to provide adequate power supply to meet the demand. Two methods have been implemented to perform forecasting of electricity demand, namely, regression analysis (RA) and artificial neural networks (ANNS). We aim to compare these two methods in this paper using the mean absolute percentage error (MAPE) to measure the forecasting performance. The results show that ANNs are more effective than $R A$ in longterm forecast. In addition to that, from our investigation into the effects of the inclusion of economic and social factors, such as population and gross domestic product (GDP), into the forecast, we conclude that the inclusion of economic and social factors do not improve the accuracy of the forecast of the chosen ANN model for electricity demand.
\end{abstract}

\section{INTRODUCTION}

There are several types of load forecasting based on the length of the forecast period, namely very shortterm load forecasts (a few minutes), short-term (hours or days ahead), medium-term (a few months ahead) and long term load forecasts (a few years ahead). Long-term load forecasts are primarily intended for capacity expansion, capital investment return studies, revenue analysis and fuel budgeting [1]. Therefore, having an accurate long term forecast of future electricity demand is vital for utility companies in order for them to provide adequate power supply to meet the demand with an acceptable level of power quality and reliability and also optimal operation and maintenance costs [2]. Several methods have been developed to perform accurate long term load forecasting. These methods can be classified into two categories: conventional approaches and techniques based on artificial intelligence (AI). Load forecasting approaches based on conventional methods forecast current value of a variable based on a mathematical combination of the previous values of that variable and previous or current value of other variables. For instance, the time series model of ARMA forecasts the current value of a variable by means of a linear combination of previous values of the variable, previous values of noise and current value of noise [3]. These methods have been utilized for a long time. However, some researchers claim that these methods cannot properly represent the nonlinear relationships that exist between the load and a series of factors that influence it [4]. Consequently, there is much interest in methods based on AI, either as standalone applications, such as expert systems [5] and artificial neural networks (ANNs) [1], [6], or in the form of socalled hybrid methods, a combination of methods to produce forecasts of electricity demand in recent years. Examples of the latter category include a combination of Fuzzy Logic Clustering Neural Network proposed by $\mathrm{Lu}$ et al. [7] and also an algorithm incorporating Elman and wavelet networks by Khoa and Oanh [2]. In addition to that, Khoa et al. [4] developed a hybrid method of functional-link net (FLN), multilayer network (MLN) and wavelet network (WN) algorithms applied for long-term load forecasting. ANNs are particularly attractive and popular, as they have the ability to learn the nonlinear relationships between load and the factors affecting it directly from historical data without the need to provide a predetermined formula to analyze the data.

This paper attempts to compare the performance between RA and a type of ANN, namely multilayer perceptron with backpropagation algorithm (MLP) network. Both models are chosen in this study due to their widespread use in the field of long term load forecasting. This paper will be divided into several sections. Section II will provide a basic description of RA and MLP, while section III will describe in detail 